\title{
Effects of connected and autonomous vehicle merging behavior on mainline human-driven vehicle
}

\author{
Lishengsa Yue, Mohamed Abdel-Aty and Zijin Wang \\ Department of Civil, Environmental, and Construction Engineering, University of Central Florida, Orlando, Florida, USA
}

\begin{abstract}
Purpose - This study aims to evaluate the influence of connected and autonomous vehicle (CAV) merging algorithms on the driver behavior of human-driven vehicles on the mainline.

Design/methodology/approach - Previous studies designed their merging algorithms mostly based on either the simulation or the restricted field testing, which lacks consideration of realistic driving behaviors in the merging scenario. This study developed a multi-driver simulator system to embed realistic driving behavior in the validation of merging algorithms.

Findings - Four types of CAV merging algorithms were evaluated regarding their influences on driving safety and driving comfort of the mainline vehicle platoon. The results revealed significant variation of the algorithm influences. Specifically, the results show that the reference-trajectory-based merging algorithm may outperform the social-psychology-based merging algorithm which only considers the ramp vehicles.

Originality/value - To the best of the authors' knowledge, this is the first time to evaluate a CAV control algorithm considering realistic driver interactions rather than by the simulation. To achieve the research purpose, a novel multi-driver driving simulator was developed, which enables multi-drivers to simultaneously interact with each other during a virtual driving test. The results are expected to have practical implications for further improvement of the CAV merging algorithm.
\end{abstract}

Keywords Driving simulator, Connected and autonomous vehicle, Merging algorithm, Merging behavior, Safety and comfort, Driving safety, Driving comfort

Paper type Research paper

\section{Introduction}

Connected and autonomous vehicle (CAV) technology has been gaining more and more attention in recent years; it releases drivers from heavy driving tasks and avoids driver errors. One challenge of CAV technology is its adaptability in critical traffic scenarios. A typical critical scenario is the merging scenario at the freeway ramp area; it is the hotspot of traffic crashes. In total, $18 \%$ of all interstate freeway crashes, $17 \%$ of the injury crashes and $11 \%$ of the fatal crashes occurred at interchanges, and most proportion of these crashes took place at the entrance or exit ramps (Ahammed et al., 2008; McCartt et al., 2004). Given that there is usually significant vehicle interaction at the merging areas, the design of the CAV merging algorithm is critically important; the algorithm is supposed to ensure a safe merging behavior; meanwhile, it is expected to disturb the mainline driving as little as possible.

Many studies have designed CAV merging control algorithms. Generally, the merging algorithms can be categorized into two types: physical-restriction-based

The current issue and full text archive of this journal is available on Emerald Insight at: https://www.emerald.com/insight/2399-9802.htm

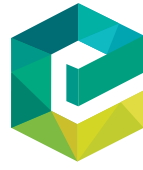

Journal of Intelligent and Connected Vehicles $5 / 1(2022) 36-45$

Emerald Publishing Limited [ISSN 2399-9802] [DOI 10.1108/JICV-08-2021-0013] merging path generation algorithm and social-psychologybased merging algorithm. For the first type, the objective of this type of merging control is to find a reference trajectory to guide the autonomous vehicle by considering physical and kinematic restrictions to fulfill a successful merging. Lu and Hedrick (2003) and Lu et al.(2004) designed a set of kinematical restriction functions in terms of the acceleration and position, to ensure that the vehicles can reach the merging points at an appropriate time. Wang et al. (2013) proposed a cooperative driving algorithm based on vehicular operation characteristics for the ramp merging. They considered the position and speed requirements for both one and two vehicles on the ramp. The second type considers driving preference regarding the merging behavior

(C) Lishengsa Yue, Mohamed Abdel-Aty and Zijin Wang. Published in fournal of Intelligent and Connected Vehicles. Published by Emerald Publishing Limited. This article is published under the Creative Commons Attribution (CC BY 4.0) licence. Anyone may reproduce, distribute, translate and create derivative works of this article (for both commercial and non-commercial purposes), subject to full attribution to the original publication and authors. The full terms of this licence maybe seen at http:// creativecommons.org/licences/by/4.0/legalcode

The authors acknowledge the financial support of the Innovative Technology Administration of US Department of Transportation, Award No. DTRT13-G-UTC53 (SAFER-SIM).

Received 19 August 2021

Revised 18 October 2021

8 November 2021

Accepted 23 November 2021 
and manipulates the merging model based on factors such as desired gap distance and desired mainstream speed. Basically, this type of algorithm defines the model in a form of preferred and actual accelerations and distance gap maintain (Awal et al., 2013a, 2013b; Chou et al., 2016; Karimi et al., 2020; Wu et al., 2019). An advanced form of the second type is to consider multiple optimization targets and generate the reference merging path by solving the optimal solution. Ding et al. (2019) proposed a rule-based merging algorithm with minimizing travel time and delay as the target; they formed a closed-form analytical solution to achieve a near-optimal merging sequence. Letter and Elefteriadou (2017) presented a longitudinal freeway merging control algorithm, which used the average travel speed as the optimization target, and they used LINGO to resolve the optimal solution.

The verification of the above algorithms is usually based on simulation platforms, either through a single platform or a cosimulation based integrated simulation platform. Some single platforms are basically microscopic traffic flow simulation software (TFSS) such as Vissim and SUMO; they have embedded driving behavior models, which can mimic carfollowing or lane change behaviors. For example, Vissim used a rule-based algorithm to initiate lateral lane change behavior and a psychophysical model for the longitudinal car-following movement (Fellendorf and Vortisch, 2001). Different from Vissim, SUMO used a Krauss model as its default carfollowing model (Bieker-Walz et al., 2017). However, individual vehicle dynamics modeling is not a strength for these platforms. On the contrary, some other single platforms, such as CarMaker, have a better ability of modeling vehicle dynamic details such as powertrain and sensor system. The driver behavior model used in the CarMaker is based on a proportional-integral-derivative controller, with considerations of psychological studies and measurements from real test drivers (Olofsson and Pettersson, 2015). Whatever driver behavior models they are, the models result from speed, speed difference, distance gap, vehicle dynamic restrictions or individual driver characteristics.

Integrated simulation platforms provide more explicit simulation regarding individual vehicle dynamics or a better power to optimize CAV control algorithms during the running time; the integrated platform can consist of a TFSS and several other simulators such as IPG CarMaker and Matlab/SIMULINK. Basically, the TFSS is more efficient at simulating microscopic traffic flow while it ignores the vehicle dynamic details; on the contrary, the IPG CarMaker can better simulate the vehicle dynamics such as the powertrain system and sensor system; while the Matlab/ SIMULINK is mainly for algorithm optimization purpose, its embedded mathematical toolboxes can be used to resolve optimization problems and generate optimized parameters/ outputs of control algorithms. When complicated traffic flow simulation is not necessary, IPG CarMaker is often used to verify CAV control algorithms, such as longitudinal cruise control (Kuutti et al., 2019), lateral lane change (Samiee et al., 2016), overtaking path planning (Nguyen et al., 2017) and tactical behavior planning (Sefati et al., 2017); while the CAV algorithm is required to be tested in certain traffic flow conditions, a co-simulation between
CarMaker, TFSS and Matlab/SIMULINK is often used (Madhusudhanan, 2019; Nalic et al., 2020a; Nalic et al., 2020b).

However, the lack of realistic driver behavior in the algorithm validation deteriorates the credibility of the algorithm. As pointed by Aksjonov et al. (2020), purely computation simulation does not guarantee realistic environments for a testing vehicle, and this is the reason that in recent years the concept of "hardware-in-the-loop" or "human-in-the-loop" becomes more prevalent (Aksjonov et al., 2020; Artunedo et al., 2015; Dang et al., 2020; Schreiber et al., 2018). Basically, using real hardware or a driver to test the algorithm is more reliable than using a driver behavior model particularly when it is necessary to observe vehicle interactions and possible improper driving adaptation behaviors such as driving errors and aggressive driving. Theoretically speaking, a driver behavior model is controlled by many kinematical restrictions with the purpose of generating "smooth" behaviors, and it is hard to mimic improper driving adaptations (Nalic et al., 2020a).

Regarding the CAV merging control algorithms, most of the aforementioned studies were based on simulation, which is hard to represent realistic driver behaviors in the merging scenario. Very few of them conducted field testing; however, because of the safety consideration, only conservative algorithms and restricted testing conditions (such as low driving speed) were tested. Many studies proved that driver behavior can significantly affect the crash and safety level at the merging area. Weng et al. (2015) found that the drivers' merging behavior is highly correlated with the rear-end crash risk; there will be high rear-end crash risks when the merging vehicle travels at either a very high or low speed. Weng and Meng (2014) found that if the merging action initiates earlier, there will be a lower rear-end crash potential. Reinolsmann et al. (2019) also suggested earlier lane change because it can contribute to smooth maneuvers and gradual speed reductions particularly at the rural expressway ramp area. Moreover, Potzy et al. (2019) concluded that drivers on the mainline prefer an efficient lane change of the autonomous vehicle on the ramp, and results show that drivers would tolerate less compliance with safety distance to have less interacting traffic. It is quite necessary to consider the realistic driver behavior for CAV merging control algorithms so that the CAV merging behavior can be more acceptable and predictable for mainline drivers.

Therefore, the objective of this study is to evaluate the influence of CAV merging algorithms on driver behavior of human-driven vehicles on the mainline, by using the human-inthe-loop concept. Several classical merging algorithms were tested in this study, representing the physical-restriction-based merging path generation algorithm and social-psychologybased merging algorithm; then their influence on the mainline traffic was analyzed given their algorithm framework features. This study is expected to conclude design principles of merging algorithms that have less influence on the mainline traffic. The driving safety and driving comfort of mainline human drivers would be analyzed to distinguish the performance of different merging algorithms. To account for crash risks in a realistic field testing, a driving simulator experiment would be used instead.

This study is organized as follows: CAV Merging Algorithm section 2 introduces classical CAV merging algorithms that 
were tested in this study; Experimental Design section 3 presents the multi-driver simulator system developed for this study, experimental scenarios and data analysis method; Results section 4 presents the results and Discussion section 5 investigates the results; finally, the Limitation section 6 presents the study limitation and Conclusion section 7 summarizes this study.

\section{Connected and autonomous vehicle merging algorithm}

Almost all CAV merging algorithm designs adopt a concept of "virtual platoon," with a connotation of projecting the CAV's position from the merging ramp to the mainline, and generating a "virtual platoon" consists of both human-driven vehicles (yellow) and projected CAV (gray) (Figure 1). The projection is exactly based on geometric parameters of the CAV, and it determines the relative position of the projection vehicle to other mainline vehicles. The core idea of the CAV merging algorithm is to manipulate the speed and acceleration of the projected $\mathrm{CAV}$, so that it can maintain a safe headway distance to the front vehicle under a desired traveling speed. Basically, in a fully connected and automated environment, a central controller will be set up covering the upstream and downstream of merging area, and collect speed and location information of all vehicles (both on mainline and ramp) entering the control area; the vehicles in the control area will be manipulated so that the speed and headway distance of each single vehicle in the virtual platoon can be well accommodated. Specifically, the controller will accommodate the autonomous vehicle (projected) based on its relative speed and position to the leading vehicle (first vehicle in the platoon), and consecutively accommodate the second and third vehicles based on similar safety considerations toward the vehicles in front of them.

In this study, the driving environment is partially connected, and only the CAV can manage its movement by sensing the leading vehicle; for the second and third vehicles, drivers need to determine the driving by themselves rather than follow the central controller. Therefore, the controller, which is embedded with the merging algorithm, will take the information of the first vehicle as input to manage the movement of the CAV.

Two types of classical CAV merging algorithms, the reference-trajectory-based merging algorithm generation algorithm and the social-psychology-based merging algorithm, were reproduced in this study based on previous studies. The study verified their effects on the vehicle platoon on the mainline. The third type of CAV merging algorithm that is

Figure 1 Virtual platoon and projected CAV

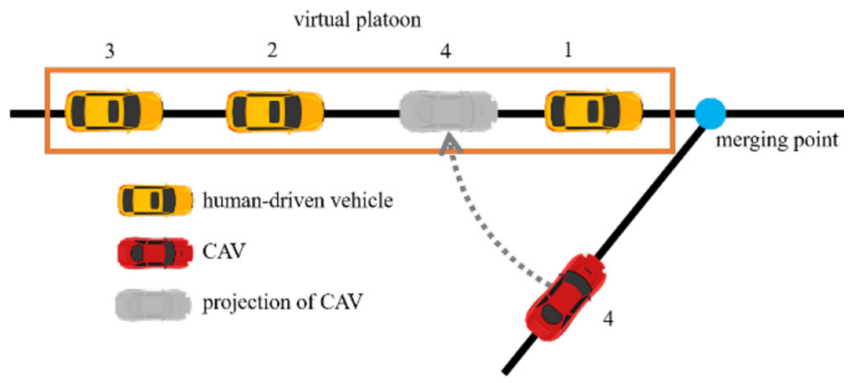

based on the optimization model was not investigated in this study, because of its computation cost in the real-time driving simulator system. These tested merging algorithms and their examples are listed below.

Reference-trajectory-based merging algorithm. This study adopted the algorithm (denoted as AHS) and its parameters proposed by Lu and Hedrick (2003) and Lu et al. (2004) as an example, which defined the reference trajectory $v_{m d}(t)$ as follows:

$$
\begin{aligned}
& v_{\text {md }}\left(t_{i}\right)=\left\{\begin{array}{c}
v_{\text {md }}\left(t_{\text {merg }}\right)=v\left(t_{\text {merg }}\right) \\
\left(1-\alpha\left(t_{i}\right)\right) v\left(t_{\text {merg }}\right)+\alpha\left(t_{i}\right) v_{p}\left(t_{i}-1\right) \\
\text { when } t_{\text {merg }} \leq\left(t_{\text {merg }}+i \Delta t\right) \leq T_{\text {virt }} ; \\
v_{p}\left(t_{i}-1\right) \\
\text { when } T_{\text {virt }}<\left(t_{\text {merg }}+i \Delta t\right) \leq T_{\text {merg }} ;
\end{array}\right. \\
& \alpha\left(t_{i}\right)=\alpha_{0}^{\beta}\left(t_{i}\right), \quad \beta>0 \\
& \alpha_{0}\left(t_{i}\right)=\frac{\sum_{j=1}^{i} v_{p}\left(t_{j}-1\right) \Delta t}{\sum_{j=1}^{i} v_{p}\left(t_{j}-1\right) \Delta t+\text { dist_para }_{\text {_pa }}}
\end{aligned}
$$

where $v(t)$ is the merging vehicle speed, $v_{p}(t)$ is the speed of the first vehicle in the platoon on mainline, $t_{\text {merg }}$ is the time when the merging algorithm starts, $T_{\text {virt }}$ is the time when the virtual platoon is established but merging is not complete yet, $T_{\text {merg }}$ is the time when the merging is finished, $\beta$ is a coefficient and dist_para is the initial distance relationship between vehicles considering desired distance before and after the merging. Detailed variable definitions can be found in $\mathrm{Lu}$ and Hedrick (2003) and Lu et al. (2004).

Social-psychology-based merging algorithm. This study tested three examples, which were borrowed from car-following models, by considering the driver's desire to main a certain speed and distance to the leading vehicle. The intelligent driver model (IDM) (Treiber et al., 2000), the generalized force model (GFM) (Helbing and Tilch, 1998) and the k-leader fuelefficient (KLFE) model (Awal et al., 2013a, 2013b) were used as examples in this study. The model parameters were identical to the ones in corresponding studies.

The IDM is given by:

$$
\begin{gathered}
a=A\left[1-\left(\frac{v}{V}\right)^{\delta}-\left(\frac{S_{\text {desire }}(v, \Delta v)}{S}\right)^{2}\right] \\
S_{\text {desire }}(v, \Delta v)=g_{0}+g_{1} \sqrt{\frac{v}{V}}+v T+\frac{v \Delta v}{2 \sqrt{A b}}
\end{gathered}
$$

where $a$ is the suggested acceleration; $v$ and $V$ are the current speed and desired speed, respectively; $\Delta v$ is the speed difference to the preceding vehicle; $S$ and $S_{\text {desire }}$ are the current following distance and desired following distance, respectively; $A$ and $b$ are maximum desired acceleration and deceleration, respectively; $g_{0}$ and $g_{1}$ are different jam distance parameters and $\delta$ is a constant coefficient. Detailed variable definitions can be found in Treiber et al. (2000).

The GFM is given by: 


$$
\begin{gathered}
a_{n}=\frac{V\left(1-e^{-\frac{s_{n-s_{\text {desire }}(v)}}{R_{a}}}\right)-v}{\tau_{a}}-b \\
b=\frac{\theta(\Delta v) \Delta v e^{-\frac{s_{n-s_{\text {desire }}(v)}}{R_{d}}}}{\tau_{d}} \\
S_{\text {desire }}(v)=s_{0}+v T
\end{gathered}
$$

where $a_{n}$ is the $n$th vehicle's acceleration; $v$ and $V$ are the current speed and desired speed, respectively; $\Delta v$ is the speed difference to the preceding vehicle; $s_{n}, s_{\text {desire }}$ and $s_{0}$ are the current following distance, desired safe following distance and minimum following distance, respectively; $\theta$ is the Heaviside function; $T$ is the safe time headway; $\tau_{a}$ and $\tau_{d}$ are the acceleration time and braking time, respectively; $R_{a}$ and $R_{d}$ are the range of the acceleration and range of the braking interaction, respectively. Detailed variable definitions can be found in Helbing and Tilch(1998).

The KLFE is given by:

$$
\begin{aligned}
& v_{n}(t+\Delta t)=\min \left[v_{n, m}(t+\Delta t)\right] \\
& v_{n, m}(t+\Delta t)=\max \left(0, \min \left(v_{n, m}^{a}(t), v_{n, m}^{\text {safe }}(t)\right)\right) \\
& v_{n, m}^{a}(t)=v_{n}(t)+k \\
& k=A \Delta t\left[1-\left(\frac{v_{n}(t)}{V}\right)^{4}-\left(\frac{v_{n}(t) s_{n, m}^{\text {desire }}(t)}{V_{S_{n, m}}}\right)^{2}\right] \\
& p=2\left[x_{m}(t)-x_{n}(t)-l^{\prime}\right]-v_{n}(t) \Delta t-\frac{v_{m}(t)^{2}}{b^{*}} \\
& v_{n, m}^{\text {safe }}(t)=\left\{\begin{array}{c}
b \Delta t+q \text { if } q \geq 0 \\
b \Delta t+v_{n}(t) \text { if } q<0
\end{array}\right. \\
& q=\sqrt{b^{2}(\Delta t)^{2}-b p} \\
& s_{n, m}^{\text {desire }}(t)=\left[S_{0}+\mathrm{b}\right] *(n-m) \\
& \left.\mathrm{b}=\max \left(0, v_{n}(t) T+\frac{v_{n}(t)\left\{v_{n}(t)-v_{m}(t)\right\}}{2 \sqrt{A|b|}}\right)\right]
\end{aligned}
$$

where $n$ and $m$ stand for the $n$th and $m$ th vehicles in the platoon; $s_{n, m}^{\text {desire }}$ is the desired following distance between the $n$th and $m$ th vehicles; $v_{n, m}^{a}$ is the following vehicle speed when the distance between the following and preceding vehicle is large, whereas the $v_{n, m}^{\text {safe }}$ is the following vehicle safe speed when the gap distance is small; $v_{n}, v_{m}$ and $V$ are the current following speed, current preceding speed and desired speed, respectively; $x_{n}$ and $x_{m}$ are the following and preceding vehicle positions; $l^{\prime}$ is the effective size plus a margin; $A$ is the maximum desired acceleration; $b$ is the maximum braking rate; $b^{*}$ is the estimated braking rate of the preceding vehicle; $T$ is the safe time headway; $S_{0}$ is the jam distance. Detailed variable definitions can be found in Awal et al. (2013a, 2013b). It is worth mentioning that the KLFE considers vehicles on both the ramp and mainline.

\section{Experimental design}

\subsection{Apparatus}

A multi-driver driving simulator system (Figure 2) was developed to test a vehicle platoon in a virtual driving scenario. Compared with a realistic field test, the advantage of using the driving simulator is that it can test dangerous driving scenarios without real collision risks. The simulator system designed a data collection module, a vehicle physics module, a scenario management module and a communication module. The data collection module collects driver behavior data in the scenario, such as brake, throttle, steering wheel, speed and position; the vehicle physics module simulates vehicle dynamics and related physical features, such as engine dynamics and collision effects; the scenario management module configures scenario control scripts, and it manages all types of scenario objects and their actions; as for the communication module, it connects multiple driver clients and distributes the simulation data between clients simultaneously (Figure 3).

\subsection{Merging scenario design}

This study designed a merging scenario as illustrated in Figure 4. Three human-driven vehicles (the first to third yellow vehicles) are traveling on the mainline, and they form a stable vehicle platoon. A CAV (fourth red vehicle) is merging into the mainline from a ramp, and it is supposed to cut in between the first and second vehicles in the platoon; the CAV is controlled by the automatic merging algorithm. The second humandriven vehicle determines whether to yield to the CAV, based on the safety consideration. Normally, the CAV would appear ahead in the second human-driven vehicle's view; therefore, the second human-driven vehicle would slow down. However, in rare cases, the second human-driven vehicle decides to accelerate and overtake the CAV; in these cases, the merging algorithm will recognize that the second human-driven vehicle would arrive at the merging point before the CAV, thus the algorithm will change its goal to following the second humandriven vehicle. The study arranged a fifth environmental vehicle (blue) in front of the vehicle platoon in the mainline; the vehicle follows a predefined path and speed, and it is used as a

Figure 2 Multi-driver driving simulator system framework

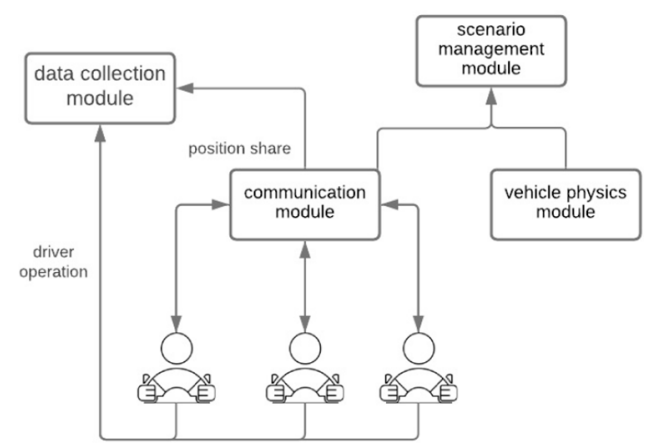


Figure 3 Multi-driver driving simulator system and experiment

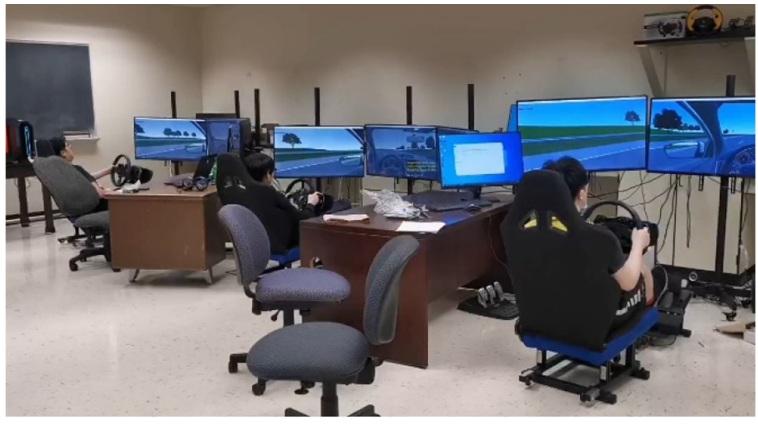

(a)

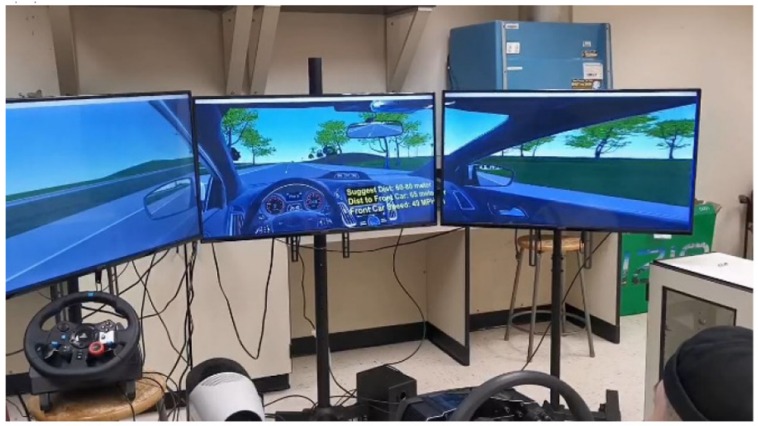

(b)

Notes: (a) Multi-driver was simultaneously connected;

(b) scenario screenshot of one driver

reference vehicle to control the speed of the vehicle platoon, in case either too fast or too slow.

In each experiment, a set of three connected drivers drove through a track that contains five merging scenarios. These five scenarios have the same merging algorithms, and the driver's average driving performance was analyzed later. The experiment was a within-subjects experiment, and three drivers experienced all five merging algorithms; therefore, in total, five experiments were conducted. The merging algorithms were presented in a randomized way to account for the order effect (Yue et al., 2020). In the experiment, the distance to the front vehicle and its speed information was displayed on the following vehicle's screen; referring to this information display, each driver was asked to follow the front vehicle keeping a distance of $60-80 \mathrm{~m}$; the speed limit was $55 \mathrm{mph}$.

\subsection{Experimental procedure}

In total, 16 groups of drivers (i.e. 48 drivers) conducted the experiment. They were driving on the mainline and an autonomous car controlled by the merging algorithm was merging from the ramp. Before the formal experiment, each

Figure 4 Merging scenario in experiment

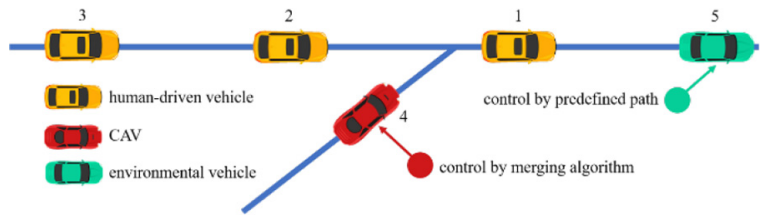

group of drivers was given a practice driving so that they can be familiar with the driving environment. During the formal experiment, each group drove four tracks, and each track had one type of merging algorithm repeated six times. In other words, the drivers experienced five merging scenarios in one track.

\subsection{Influence period during merging}

It is worth mentioning that during the above "merging scenario," the CAV and second vehicle would not interact with each other all the time. Because of the sight of the second driver's view, only when the CAV is close enough, the second driver would be affected and accommodate driving behaviors to the merging vehicle. This period is defined as the influence period.

Given that the driving behaviors are assumed to be different between the normal and influenced driving periods, the finite Gaussian mixture model (GMM) was used to distinguish two periods in the merging scenario. The GMM assumes that data of different features is coming from a mixture of two or more Gaussian distributions (i.e. clusters), and the GMM allocates data points to most probable distributions by expectationmaximization (EM) algorithm (Scrucca et al., 2016). Similar trajectory clustering practice was conducted by Mohammed et al.(2019); the researchers used finite GMM to cluster cyclists overtaking and following trajectories into different states.

In the merging scenario, the second driver manages the throttle and brake to maintain the safety buffer between both the first vehicle and CAV. Therefore, this study used second vehicle's speed, throttle and brake positions and distances to the first vehicle and CAV as trajectory features to be clustered. Figure 4 shows an example of the throttle clusters generated by GMM. It shows that at the time of around 100, the second driver notices the merging $\mathrm{CAV}$ and begins to monitor the collision risk; then at the time of around 160, the second driver begins to release the pedal position to slow down; during the time point of 100-160, the throttle position does not change, this might be because of the driver's reaction time delay. It is worth mentioning that the driving periods of the second driver were applied to the third driver in this study (Figure 5).

\subsection{Driving performance metrics}

This study mainly investigated the influence of CAV merging algorithms on the second and third human-driven vehicles. Two driving periods were analyzed: the merging and following periods. The merging period is defined as the period from the

Figure 5 Throttle position during merging scenario

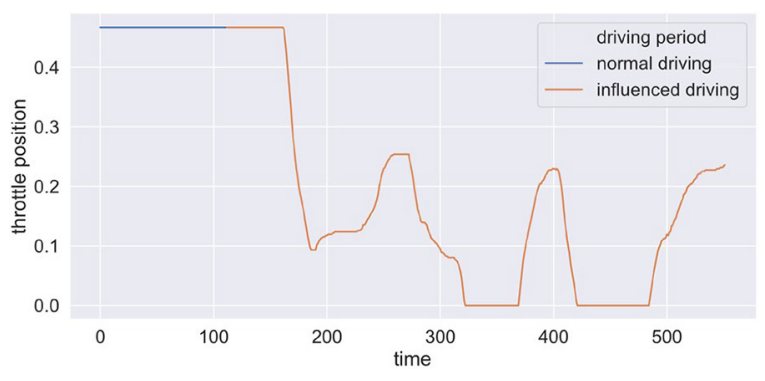


time the CAV begins to move on the ramp to the time it arrives at the merging point, which occurs in the merging scenario.

The following period (Figure 6) is from the time that the CAV finishes merging and begins to drive with the vehicle platoon, to the time that the CAV leaves the lane of vehicle platoon (the leaving point was given); the following actions specifically refer to the actions of second human-driven vehicle (following the CAV) and third human-driven vehicle (following the second human-driven vehicle).

For each driving period, two aspects of metrics were collected in terms of both driving safety and driving comfort. The driving safety measures include the minimum time-tocollision (TTC) and the deceleration rate to avoid crashes (DRAC). The TTC was introduced by Hayward(1972) and is the most widely used surrogate safety measure (SSM); it indicates the time that is left for the following vehicle to hit a leading vehicle. The TTC is given by:

$$
T T C=\left\{\begin{array}{c}
\frac{S-L}{v_{2}-v_{1}}, v_{2}>v_{1} \\
\infty, \text { otherwise }
\end{array}\right.
$$

where $S$ is the distance gap between the leading and following vehicles; $L$ is the vehicle length; $v_{2}$ and $v_{1}$ are speeds of following and leading vehicles, respectively. TTC can be calculable only when $v_{2}$ is greater than $v_{1}$. In this study, TTC was assigned a large value of 100 when $v_{2}<v_{1}$. Obviously, a large value of minimum TTC indicates a high safety level.

The DRAC is also a widely used SSM, proposed by Cooper and Ferguson (1976); it indicates the required minimum deceleration rate for a following vehicle to avoid a crash with a leading vehicle. The DRAC is given by:

$$
D R A C=\left\{\begin{array}{c}
\frac{\left(v_{2}-v_{1}\right)^{2}}{S-L}, v_{2}>v_{1} \\
0, \text { otherwise }
\end{array}\right.
$$

Usually, a threshold $D R A C^{*}$ would be selected and the percentage of DRAC $>$ DRAC $^{*}$ is used to indicate the safety level; a large percentage represents a dangerous situation. In this study, a threshold DRAC* of $3.0 \mathrm{~s}$ was adopted, and the "DRAC" term mentioned in later sections is the percentage value, weighted by trajectory length. Both the minimum TTC and DRAC were applied to the merging and following periods.

In terms of the driving comfort measures, the mean deceleration/acceleration and the average jerk during the deceleration/acceleration processes were used for the merging period. The jerk is the derivative of deceleration/acceleration. In the merging period, these measures were proved to have a negative relationship with driving comfort (Bellem et al., 2016, 2018;

Figure 6 Following period when a CAV cuts in between the first and second vehicles

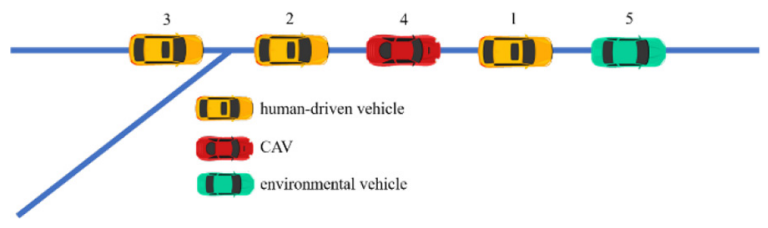

Nandi et al., 2015). In the following period, the minimum headway distance was used to indicate the driving comfort level (Bellem et al., 2016); the larger the headway distance, the more comfortable the driving is.

\section{Results}

\subsection{Influence on driving safety}

During the merging period, for the second driver, both the minimum TTC and DRAC between the four merging algorithms were significantly different $(F=5.62$, $p$-value $<$ 0.01 and $F=14.45, p$-value $<0.01$, respectively). Figure 7 shows that the AHS algorithm had the largest minimum TTC, which was $19.45 \mathrm{~s}$; significantly smaller than the AHS algorithm, the GFM algorithm had a minimum TTC of only $6.49 \mathrm{~s}$. As for the IDM and KLFE algorithms, their minimum TTC values were between AHS and GFM; the KLFE had a slightly higher safety level than the IDM. In addition, the AHS algorithm had a very small DRAC of only 0.007; the KLFE's DRAC was slightly larger, i.e. 0.009, whereas the GFM and IDM had a much larger DRAC of around 0.7. For the third driver, only the minimum TTC was found significantly different between merging algorithms $(F=2.45$, $p$-value $=$ 0.056). Similar to the influence on the second driver, the AHS had the largest minimum TTC, which was 36.77 s; the GFM had the smallest minimum TTC of only $22.16 \mathrm{~s}$. The level of minimum TTC of the IDM and KLFE was between AHS and GFM.

During the following period, the second driver's minimum TTC was found significantly different between four types of merging algorithms $(F=2.31, p$-value $=0.068)$. The AHS had the largest minimum TTC of $41.30 \mathrm{~s}$, whereas the KLFE had the smallest minimum TTC of 30.23 s. The GFM was slightly larger than KLFE, which was 36.20 s, and the IDM had a minimum TTC of 38.40 s. Similar to the second driver, the third driver had significantly different minimum TTC between merging algorithms $(F=3.81, p$-value $<0.01)$. Specifically, the AHS had the largest minimum TTC of $11.61 \mathrm{~s}$, whereas the GFM and IDM had the smallest minimum TTC of around $8.35 \mathrm{~s}$. The minimum TTC of KLFE was $9.58 \mathrm{~s}$.

\subsection{Influence on driving comfort}

During the merging period, the second driver's mean deceleration was significantly different between the four merging algorithms $(F=4.74, p$-value $=0.002)$. Figure 8 shows that the AHS had the smallest deceleration of $2.64 \mathrm{~m} / \mathrm{s}^{2}$, whereas the GFM had the largest deceleration of $4.23 \mathrm{~m} / \mathrm{s}^{2}$. The decelerations of the KLFE and IDM were in between with a value of 3.38 and $3.11 \mathrm{~m} / \mathrm{s}^{2}$, respectively. The average jerks during the deceleration process were also found significantly different between the merging algorithms $(F=14.45$, $p$-value $<$ 0.01). To be specific, the GFM had the largest average jerk during the deceleration process, which was 36.86; the IDM and AHS had a relatively smaller average jerk value close to each other of around 27.50; the jerk value of the KLFE was in between and it was 30.04. In terms of the minimum headway distance, a significant difference was found because of the merging algorithms $(F=62.26, p$-value $<0.01)$. The AHS and KLFE had a very large minimum headway distance of 37.99 and $36.11 \mathrm{~m}$, respectively, whereas the GFM had the smallest 
minimum headway distance of $17.57 \mathrm{~m}$. The IDM had a minimum headway distance of $23.64 \mathrm{~m}$, slightly larger than the GFM. For the third driver, the average jerks during the acceleration process were found significantly different between the merging algorithms $(F=2.21, p$-value $=0.08)$. The AHS had the largest average jerk of 47.80 , whereas the KLFE had the smallest average jerk of 36.83. The average jerks of the GFM and IDM were close to each with a value of 39.62 and 40.48 , respectively.

During the following period, the second driver, both the mean acceleration and deceleration were found to be significantly affected by the merging algorithm. For mean acceleration, the GFM had the largest one of $4.33 \mathrm{~m} / \mathrm{s}^{2}$, whereas the AHS had the smallest one of $3.34 \mathrm{~m} / \mathrm{s}^{2}$. The mean accelerations of IDM and KLFE were 3.79 and $3.77 \mathrm{~m} / \mathrm{s}^{2}$, respectively. Additionally, the GFM had the largest mean deceleration of $3.47 \mathrm{~m} / \mathrm{s}^{2}$, whereas AHS had the smallest mean deceleration of $2.89 \mathrm{~m} / \mathrm{s}^{2}$. The mean deceleration of the IDM and KLFE was close to each other. In terms of the average jerk, it was found to be significantly affected by the merging algorithms during the acceleration process $(F=4.92$, $p$-value $<0.01)$. The GFM had the largest one of 46.81 , whereas the AHS had the smallest one of 41.93. The average jerks of IDM and KLFE were 43.29 and $43.72 \mathrm{~m} / \mathrm{s}^{2}$, respectively. For the minimum headway distance, a significant difference was also found $(F=47.21$, $p$-value $<0.01$ ). The GFM had the smallest one of $21.60 \mathrm{~m}$, whereas the AHS had the largest one of $41.01 \mathrm{~m}$. The KLFE also had a large minimum headway distance of $40.69 \mathrm{~m}$, whereas the value of IDM was $32.07 \mathrm{~m}$.

During the following period, the third driver's mean acceleration and minimum headway distance were found to be significantly affected by the merging algorithm $(F=2.43$, $p$ value $=0.06$ and $F=4.11, p$-value $<0.01$, respectively). The GFM had the largest mean acceleration of $4.51 \mathrm{~m} / \mathrm{s}^{2}$, whereas the other three algorithms are very close. In terms of the minimum headway distance, the AHS had the largest one of $53.59 \mathrm{~m}$, whereas the other three algorithms are very close.

\section{Discussions}

The heterogeneity of CAV merging behavior caused significantly different influences on the mainline vehicle platoon. For the second driver, regarding the driving safety in either merging or following periods, the minimum TTC and DRAS show that AHS had the least negative influence, whereas the GFM had the most negative influence. Regarding the driving comfort, the deceleration, jerk and headway distance show that the AHS had the best driving comfort, whereas the GFM had the worst driving comfort in both two driving periods. The influence of IDM and KLFE on driving safety and comfort was generally in between the AHS and GFM. Compared with the second driver, for the third driver, the heterogeneity of influence of CAV merging behaviors was much less significant. In terms of driving safety, the DARC was not significantly different and only the minimum TTC was observed to be varied between CAV algorithms. The minimum TTC shows that in both merging and following periods, the AHS was the safest, whereas the GFM was the riskiest one. In terms of driving comfort, in the merging period, the average jerk shows that the AHS was the least comfortable one, and both the GFM and IDM had a better comfortable level than the AHS; the KLFE was the most comfortable one. In the following period, the mean acceleration shows that the GFM was the least comfortable one, whereas the minimum headway distance shows that the AHS is the most comfortable one.

The results show that the AHS can guarantee driving safety and driving comfort among the tested four types of merging algorithms; nevertheless, its driving comfort may deteriorate for the later part of the vehicle platoon in the

Figure 7 Driving safety level of automatic merging algorithm

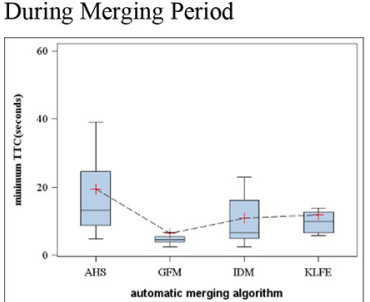

(a)

During Following Period

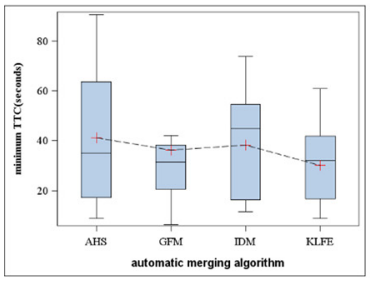

(d)

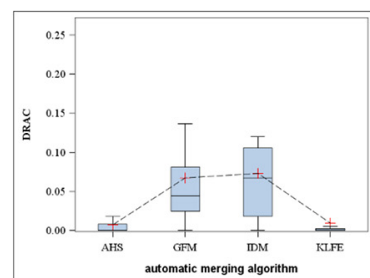

(b)

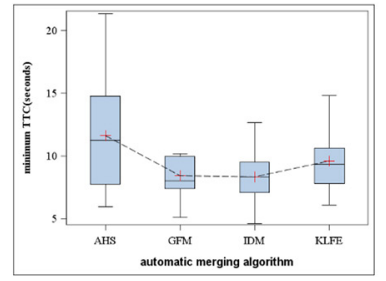

(e)

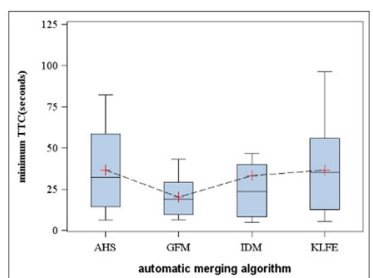

(c)

Notes: During Merging Period; (a)minimum TTC-2nd driver; (b)DRAC-2nd driver; (c)minimum TTC-3rd driver; During Following Period; (d)minimum TTC-2nd driver; (e) minimum TTC-3rd driver 
merging period. It was interesting that the AHS, as a reference-trajectory-based merging algorithm, can achieve a comprehensive good performance in terms of both driving safety and comfort, without considering many other optimization goals. In addition, the KLFE maybe the second-best merging algorithm. The KLFE is a socialpsychology-based merging algorithm; different from the GFM and IDM, it additionally considers fuel efficiency in its model. It seems that among social-psychology-based merging algorithms, the one that considers more factors can achieve a better influence on the vehicle platoon on the mainline. This might be because the merging driver's social-psychology desire as well as additional optimal targets for the mainline traffic were both considered. The
GFM was the worst merging algorithm because it caused more negative effects on driving safety and driving comfort; this might be because it is not much suitable to capture a driver's comprehensive driving preference.

\section{Limitations}

The selected algorithms are not the most recent; nevertheless, they are very classical and representative that many more advanced algorithms developed their framework based on the extension of these classical algorithms. Given that it is hard to evaluate all merging algorithms, the evaluation on classical algorithms would be a more practical way. While that the four algorithms selected in the research

Figure 8 Driving comfort level of automatic merging algorithm

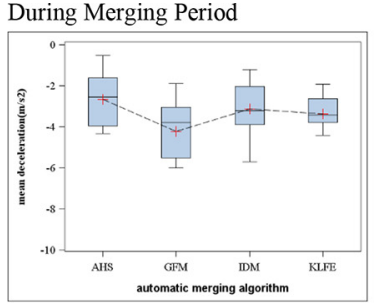

(a)

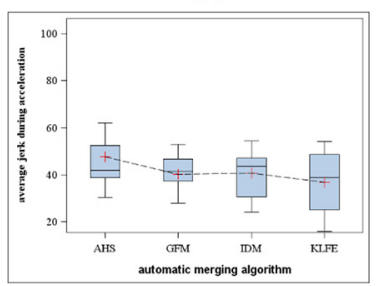

(d)

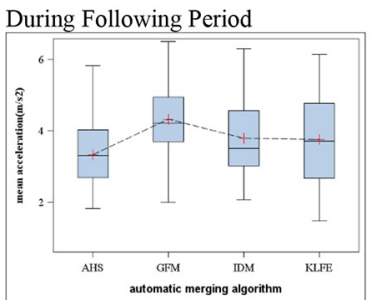

(a)

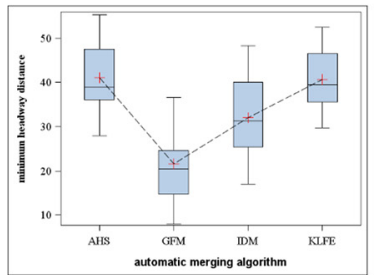

(d)

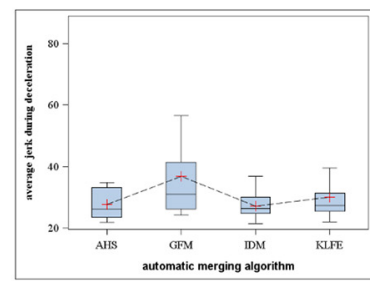

(b)

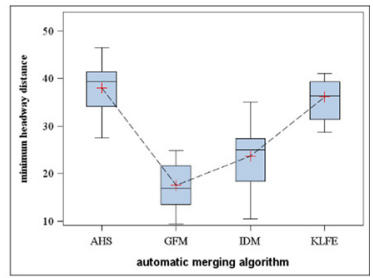

(c)

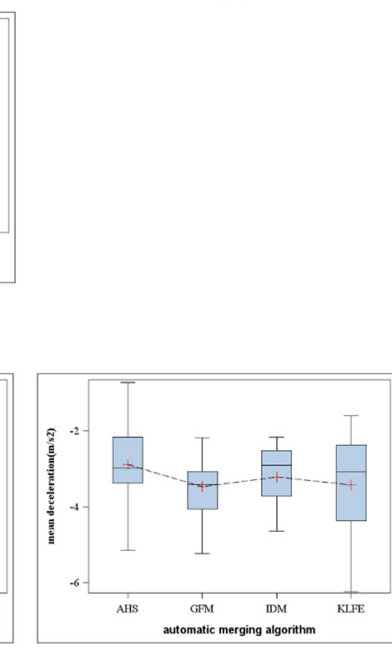

(b)

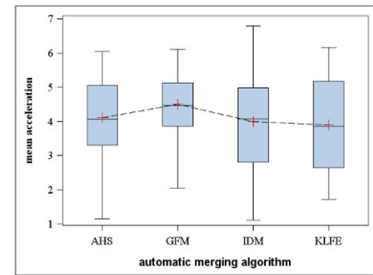

(e)

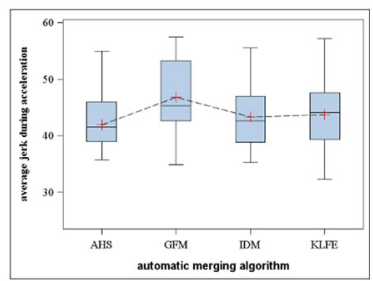

(c)

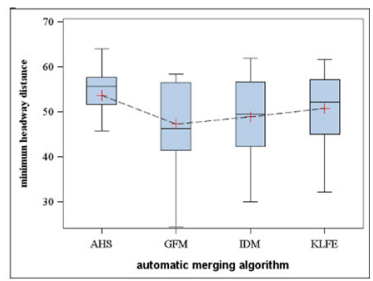

(f)

Notes: During Merging Period; (a)mean deceleration-2nd driver; (b)average jerk during deceleration process-2nd driver; (c) minimum headway distance -2nd driver; (d) average jerk during acceleration process-3rd driver; During Following Period; (a)mean acceleration-2nd driver; (b) mean deceleration-2nd driver; (c) average jerk during acceleration process-2nd driver; (d) minimum headway distance-2nd driver; (e) mean acceleration-3rd driver; (f) minimum headway distance-3rd driver 
may not be enough to represent the two categories of the merging algorithms, more merging algorithms should be added to evaluate in the future.

\section{Conclusions}

This study analyzed CAV merging behaviors' influence on the mainline vehicle platoon considering driving safety and comfort, by considering the merging algorithm heterogeneity. Four types of merging algorithms, including the referencetrajectory-based merging algorithm and the social-psychologybased merging algorithm, were evaluated by using a selfdeveloped multi-driver simulator system. The results show that although these algorithms achieved good performance in their original simulation studies, their effects were quite various when the realistic driving behavior was embedded into the experiment. The results show that the reference-trajectorybased merging algorithm may outperform the socialpsychology-based merging algorithm which only considers the ramp vehicle. More types of CAV merging algorithms need to be verified and compared in the future to form a solid conclusion.

\section{References}

Ahammed, M.A., Hassan, Y. and Sayed, T.A. (2008), "Modeling driver behavior and safety on freeway merging areas”, fournal of Transportation Engineering, Vol. 134 No. 9, pp. 370-377.

Aksjonov, A., Ricciardi, V., Augsburg, K., Vodovozov, V. and Petlenkov, E. (2020), "Hardware-in-the-loop test of an open-loop fuzzy control method for decoupled electrohydraulic antilock braking system", IEEE Transactions on Fuzzy Systems, Vol. 29 No. 5, pp. 965-975.

Artunedo, A., Godoy, J., Haber, R., Villagrá, J. and del, T.,R. M. (2015), "Advanced co-simulation framework for cooperative maneuvers among vehicles", 2015 IEEE 18th International Conference on Intelligent Transportation Systems. IEEE, pp. 1436-1441.

Awal, T., Kulik, L. and Ramamohanrao, K. (2013a), "A kleader fuel-efficient traffic model”, 2013 IEEE Intelligent Vehicles Symposium (IV). IEEE, pp. 1014-1021.

Awal, T., Kulik, L. and Ramamohanrao, K. (2013b), “Optimal traffic merging strategy for communication-and sensorenabled vehicles", 16th International IEEE Conference on Intelligent Transportation Systems (ITSC 2013). IEEE, pp. 1468-1474.

Bellem, H., Schönenberg, T., Krems, J.F. and Schrauf, M. (2016), "Objective metrics of comfort: developing a driving style for highly automated vehicles", Transportation Research Part F: Traffic Psychology and Behaviour, Vol. 41, pp. 45-54.

Bellem, H., Thiel, B., Schrauf, M. and Krems, J.F. (2018), "Comfort in automated driving: an analysis of preferences for different automated driving styles and their dependence on personality traits", Transportation Research Part F: traffic Psychology and Behaviour, Vol. 55, pp. 90-100.

Bieker-Walz, L., Behrisch, M. Junghans, M. and Gimm, K. (2017), "Evaluation of car-following-models at controlled intersections".
Chou, F.-C., Shladover, S.E. and Bansal, G. (2016), "Coordinated merge control based on V2V communication", 2016 IEEE Vehicular Networking Conference (VNC). IEEE, pp. 1-8.

Cooper, D.F. and Ferguson, N. (1976), "Traffic studies at TJunctions. 2: A conflict simulation record", Traffic Engineering \& Control, Vol. 17 No. Analytic.

Dang, D., Gao, F. and Hu, Q. (2020), "Motion planning for autonomous vehicles considering longitudinal and lateral dynamics coupling", Applied Sciences, Vol. 10 No. 9, p. 3180.

Ding, J., Li, L., Peng, H. and Zhang, Y. (2019), "A rule-based cooperative merging strategy for connected and automated vehicles”, IEEE Transactions on Intelligent Transportation Systems, Vol. 21 No. 8, pp. 3436-3446.

Fellendorf, M. and Vortisch, P. (2001), "Validation of the microscopic traffic flow model VISSIM in different realworld situations", Transportation Research Board 80th Annual Meeting.

Hayward, J.C. (1972), "Near miss determination through use of a scale of danger".

Helbing, D. and Tilch, B. (1998), "Generalized force model of traffic dynamics”, Physical Review E, Vol. 58 No. 1, p. 133.

Karimi, M., Roncoli, C., Alecsandru, C. and Papageorgiou, M. (2020), "Cooperative merging control via trajectory optimization in mixed vehicular traffic", Transportation Research Part C: Emerging Technologies, Vol. 116, p. 102663.

Kuutti, S., Bowden, R., Joshi, H., de Temple, R. and Fallah, S. (2019), "End-to-end reinforcement learning for autonomous longitudinal control using advantage actor critic with temporal context”, 2019 IEEE Intelligent Transportation Systems Conference (ITSC). IEEE, pp. 2456-2462.

Letter, C. and Elefteriadou, L. (2017), "Efficient control of fully automated connected vehicles at freeway merge segments", Transportation Research Part C: Emerging Technologies, Vol. 80, pp. 190-205.

Lu, X.-Y. and Hedrick, J.K. (2003), "Longitudinal control algorithm for automated vehicle merging", International fournal of Control, Vol. 76 No. 2, pp. 193-202.

Lu, X.-Y., Tan, H.-S., Shladover, S.E. and Hedrick, J.K. (2004), "Automated vehicle merging maneuver implementation for AHS”, Vehicle System Dynamics, Vol. 41 No. 2, pp. 85-107.

McCartt, A.T., Northrup, V.S. and Retting, R.A. (2004), "Types and characteristics of ramp-related motor vehicle crashes on urban interstate roadways in Northern Virginia", Fournal of Safety Research, Vol. 35 No. 1, pp. 107-114.

Madhusudhanan, A.K. (2019), "A method to improve an electric vehicle's range: efficient cruise control”, European Fournal of Control, Vol. 48, pp. 83-96.

Mohammed, H., Bigazzi, A.Y. and Sayed, T. (2019), "Characterization of bicycle following and overtaking maneuvers on cycling paths", Transportation Research Part C: Emerging Technologies, Vol. 98, pp. 139-151.

Nalic, D., Pandurevic, A., Eichberger, A. and Rogic, B. (2020b), "Design and implementation of a co-simulation framework for testing of automated driving systems", Sustainability, Vol. 12 No. 24, p. 10476.

Nalic, D., Li, H., Eichberger, A., Wellershaus, C., Pandurevic, A. and Rogic, B. (2020a), "Stress testing method for 
scenario-based testing of automated driving systems", IEEE Access, Vol. 8, pp. 224974-224984.

Nandi, A.K., Chakraborty, D. and Vaz, W. (2015), "Design of a comfortable optimal driving strategy for electric vehicles using multi-objective optimization", Fournal of Power Sources, Vol. 283, pp. 1-18.

Nguyen, N.A., Moser, D., Schrangl, P., del Re, L. and Jones, S. (2017), "Autonomous overtaking using stochastic model predictive control", 2017 11th Asian Control Conference (ASCC). IEEE, pp. 1005-1010.

Olofsson, M. and Pettersson, J. (2015), Parameterization and Validation of Road and Driver Behavior Models for Carmaker Simulations and Transmission HIL-Rig.

Potzy, J., Feinauer, S., Siedersberger, K.-H. and Bengler, K. (2019), "Manual drivers' evaluation of automated merging behavior in dense traffic: efficiency matters", 2019 IEEE Intelligent Transportation Systems Conference (ITSC). IEEE, pp. 3454-3460.

Reinolsmann, N., Alhajyaseen, W., Brijs, T., Pirdavani, A., Hussain, Q. and Brijs, K. (2019), "Investigating the impact of dynamic merge control strategies on driving behavior on rural and urban expressways - a driving simulator study", Transportation Research Part F: Traffic Psychology and Behaviour, Vol. 65, pp. 469-484.

Samiee, S., Azadi, S., Kazemi, R. and Eichberger, A. (2016), "Towards a decision-making algorithm for automatic lane change manoeuvre considering traffic dynamics", PROMETTrafficETransportation, Vol. 28 No. 2, pp. 91-103.

Schreiber, V., Ivanov, V., Augsburg, K., Noack, M., Shyrokau, B., Sandu, C. and Els, P.S. (2018), "Shared and distributed $\mathrm{X}$-in-the-loop tests for automotive systems: feasibility study", IEEE Access, Vol. 6, pp. 4017-4026.

Scrucca, L., Fop, M., Murphy, T.B. and Raftery, A.E. (2016), "Mclust 5: clustering, classification and density estimation using Gaussian finite mixture models", The R fournal, Vol. 8 No. 1, p. 289.

Sefati, M., Chandiramani, J., Kreisköther, K., Kampker, A. and Baldi, S. (2017), "Towards tactical behaviour planning under uncertainties for automated vehicles in urban scenarios", 2017 IEEE 20th International Conference on Intelligent Transportation Systems (ITSC). IEEE, pp. 1-7.

Treiber, M., Hennecke, A. and Helbing, D. (2000), "Congested traffic states in empirical observations and microscopic simulations", Physical Review E, Vol. 62 No. 2, p. 1805.

Wang, Y., Wenjuan, E., Tang, W., Tian, D., Lu, G. and Yu, G. (2013), "Automated on-ramp merging control algorithm based on internet-connected vehicles", IET Intelligent Transport Systems, Vol. 7 No. 4, pp. 371-379.

Weng, J. and Meng, Q. (2014), "Rear-end crash potential estimation in the work zone merging areas", fournal of Advanced Transportation, Vol. 48 No. 3, pp. 238-249.

Weng, J., Xue, S., Yang, Y., Yan, X. and Qu, X. (2015), "Indepth analysis of drivers' merging behavior and rear-end crash risks in work zone merging areas", Accident Analysis $\mathcal{E}$ Prevention, Vol. 77, pp. 51-61.

Wu, P., Gao, F. and Li, K. (2019), “A vehicle type dependent car-following model based on naturalistic driving study", Electronics, Vol. 8 No. 4, p. 453.

Yue, L., Abdel-Aty, M., Wu, Y., Yuan, J. and Morris, M. (2020), "Influence of pedestrian-to-vehicle technology on drivers' response and safety benefits considering pre-crash conditions", Transportation Research Part F: Traffic Psychology and Behaviour, Vol. 73, pp. 50-65.

\section{Corresponding author}

Lishengsa Yue can be contacted at: 2017lishengsa@knights. ucf.edu 